\title{
EXPLORING METHODOLOGICAL PROCEDURES OF TORC: AN APPLICATION EXPERIENCE
}

\author{
Éder Henriqson ${ }^{1}$ \\ Felipe Lando ${ }^{I}$ \\ Marina Gaspareto ${ }^{1}$ \\ 1) PUCRS (Pontifícia Universidade Católica do Rio Grande do Sul), Brazil.
}

\begin{abstract}
Training has proven to be effective in developing safety in complex systems, such as in aviation. Among recent training technologies for resilience skills and capabilities, TORC (Training for Operational Resilience Capabilities) was developed as a tool for training workers to handle critical situations. It is a non-specific domain gamification to address operational and organizational safety needs. Although it is a promising technology, some methodological procedures should be analyzed in order to promote its development and evaluation. In this study, we report our recent TORC experience with aviation pilots and we explore the methodological procedures in three phases of the game: preparation (i.e. objectives, game definitions and context), application (i.e. training process and training format) and analyses (i.e. discussion about relevance and training method). We adopted a multi-method approach, with one team of researchers taking part in TORC sessions and another team observing TORC dynamics. Data was cross-examined and a framework is proposed, which will offer a visual representation to guide the TORC sessions development. Results provide information for both researchers (concerned in the application of TORC and similar training dynamics) and practitioners (interested in TORC game).
\end{abstract}

Keywords: TORC. Resilience. Safety. Training

\section{INTRODUCTION}

The ability to respond to uncertainties by disarming, recovering or adapting to undesired and unexpected conditions in Complex Sociotechnical Systems (CSTS) is called Resilience Capability. Safety performance can be examined as a manifestation of such capabilities in the form of antecedents and determinants of system's response. Antecedents are present in the day-to-day operations and determinants emerge during critical situations.

One way to develop resilient performance in a system is through training with a focus on human factors, combining technical and non-technical competences. Training programs, such as CRM (Crew Resource Management) and TORC (Training for Operational Resilience Capabilities), can contribute to improve operational safety. They are expected to advance expertise of operators and managers by exposing them to scenarios where decisions must be taken with a specific set of resources and constraints. Participants are required to develop sensemaking and understanding of a situation they probably never faced before while mobilizing knowledge, experience and expertise in order to create new repertories [1]. Moreover, considering Hollnagel's [2] four cornerstones of resilience, we understand that

(C) 2019Authors. This is an Open Access article distributed under the terms of the Creative Commons AttributionNonCommercial 4.0 International License (http://creativecommons.org/licenses/by-nc/4.0), permitting all non-commercial use, distribution, and reproduction in any medium, provided the original work is properly cited.

ISBN: 978-91-88898-41-8

DOI: https://doi.org/10.15626/rea8.09 
training is a way of promoting organizational learning by using the system's experience to develop expertise and knowledge of resources available in case of critical situations.

In this study we applied and examined TORC (Training for Operational Resilience Capabilities, developed by Grøtan et al. [3] and Grøtan, van der Vorm and Macchi [4]) sessions with aviation pilots in order to assess its potential impact at individual and organizational level. This experience offers insights of what went well and what could be improved in terms of developing an application framework.

\section{RESILIENCE}

Resilience is something a systems does, not something a system has [5]. This assumption guides us to look at situations where resilience manifest, and these situations are, most of the time, critical unexpected situations. When facing this kind of event, knowledge and resources are allocated in order to restore operational performance.

We understand that resilience, in this sense, can be defined as a system behavior while handling critical situations. Grøtan [6] argues that this response is situated in a grey space between a fully compliant operation and a fully resilient (adaptive) one. The author argues that the more critical is the situation and the less experience, resources and systemic knowledge are available to operators, the greater is the possibility of adaptations.

Based on this dialectical view of Compliance versus Resilience, Grøtan developed a gaming tool to help operators and managers understand to what extent their system is capable of providing a suitable response to a critical situation. In this game, players are supposed to interact and create understanding of a critical situation and point in a scale how the organization is prepared to handle the situation (from R1 to R4 [5]). The authors propose that while R1 is a rule-supported response, where the system is capable to handle the situation by means of its procedures and available resources, R4 stands for a fully adaptive response, where the system does not have knowledge, rules or even resources to handle the situation and creativity has to be used in order to avoid a catastrophe.

\section{TRAINING FOR OPERATIONAL RESILIENCE CAPABILITIES}

Training for Operational Resilience Capabilities (TORC) seeks for the development of resilience principles in organizations. This training has a gaming format, been able to be applied in operational, managerial and integrated (joining of operational and managerial) levels. In this game participants are immersed in possible scenarios of their work environment in a critical context. The group should seek together to manage unexpected situations, based on their experiences and considering the existing rules and procedures in their organizations. Among TORC's objectives are: (a) enable operational personnel to understand the maneuverability limits available; (b) eliciting informal and formal forms of individual and team decision-making to deal with situations of operational demands; (c) learn what strategies and resources your company has available; (d) highlight and open the dialogue on the tensions between resilient behavior and limits of conformity; (e) train to collaborate with relevant decision makers and support staff [3] [4].

Training is offered for up to 8 participants. In addition to players, other professionals can be invited to be observers, who follow the dynamics of the game and can play a key role in the after-action review phase. In total, the training may consist of six to ten trainees.

Because it is a game, participants are challenged to solve scalable critical scenarios. Decisions are recorded, as well as strategies, resources and "investments". For each training session, a scenario is defined and each round will have an aggravating situation. In this way, the phases of the game are: (a) a scenario based on real company situations is presented; (b) for each round, a leader is defined who will lead the team and write down the decisions; (c) the leader picks a game changer, which introduces a difficulty to the game; (d) the new scenario 
should be discussed in terms of situation assessment, sense making, anticipate, decide, monitor effects and after action review; (e) in the end, a decision is made, considering strategies, resources and investments.

At the end of the match, all decisions, resources and investments are discussed in order to debrief and discuss for learning purpose.

\section{TORC GAMING APPLICATION}

We conducted two sessions of eight hours of TORC with two groups of aviation pilots under the training for Commercial Pilot License (flight hours experience ranging from 50 to 120h). The activity counted on twelve participants and six researchers (two researchers were trainers of the game and other four were observing TORC dynamics and methodological aspects). The agenda begun with a presentation of the study and TORC followed by two gameplays with each group, a debriefing with participants and a debriefing among researchers.

In the preparation phase, we created the scenarios and game changers specific to our application, which it had to make sense considering participants experiences. These scenarios were based on real stories that took place at aerodromes were pilots familiar to operations. These stories were made up of critical situations as game changers, which were added to each round, challenging players to make decisions in an increasingly complex setting. We have adapted all materials used by participants, translating the originals which were in a foreign language. We also developed a supporting material which describe the reflection in action' steps, the resources, and strategies. This material was used as a guide to the participants.

The morning schedule featured an initial presentation of a resilience overview and gaming instructions and the first game. The familiarity of the participants with the theme of resilience allowed the initial presentation to be more objective and shorter. In the afternoon was held the second game and the closing of the momentum of a reflection about the day experienced. This application showed us a set of difficulties related to the game. These constraints served as basis to the development of an application framework described furtherly. After the gaming session, participants were asked to provide feedback and this data was also used in the framework.

\section{A TORC APPLICATION FRAMEWORK}

The framework presents three phases of the TORC training: preparation (i.e. objectives, game definitions and context), application (i.e. the process and types) and analyses (i.e. discussion about relevance and about the training itself). Figure 1 shows the framework proposed. The aim of this framework is to provide a structure to TORC applications. It was developed based on discussions on each phase described, as it is explained below. 


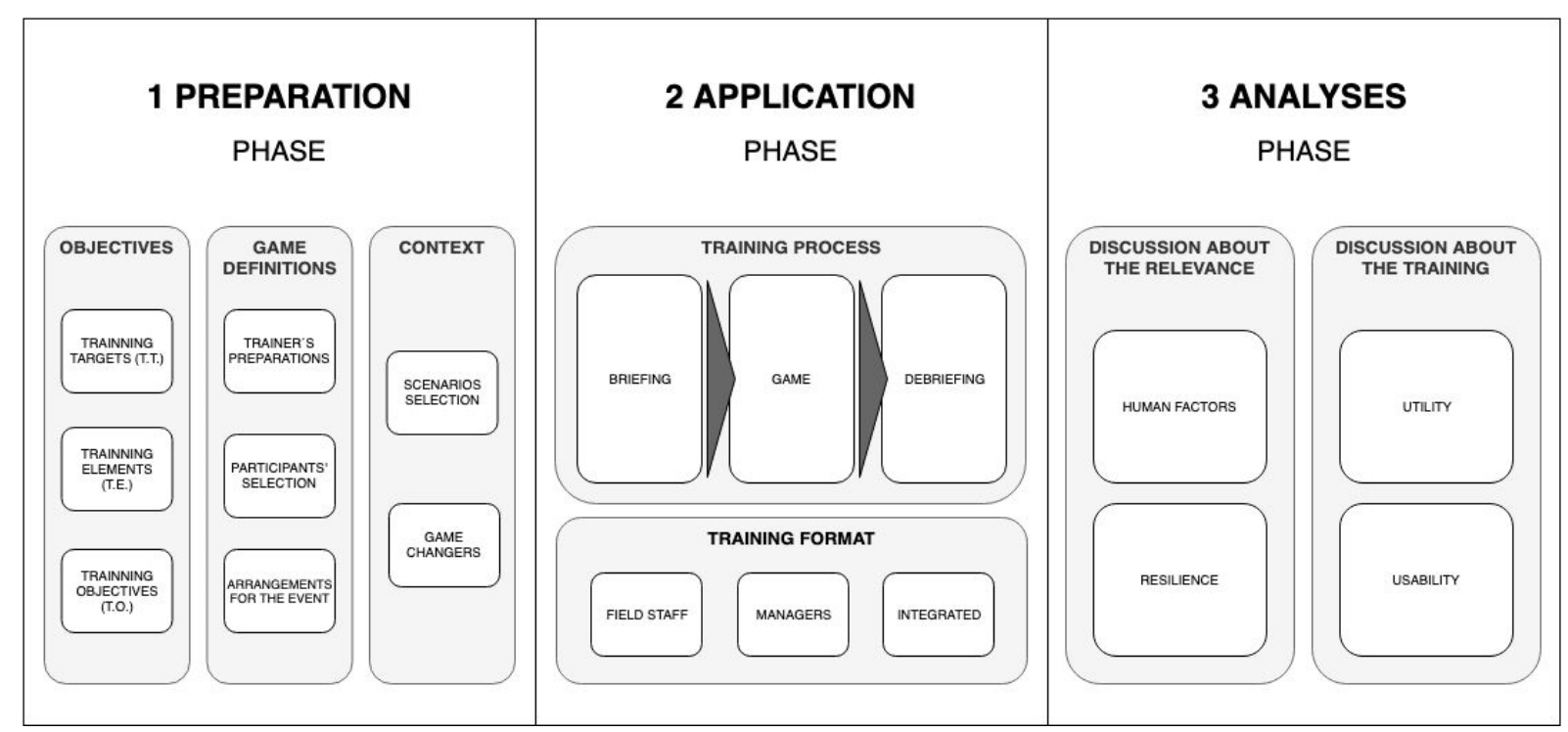

Figure 1. Application framework

\subsection{Preparation phase}

This phase consists in setting up objectives of the game, defining operational details and gaming context. The difficulty faced here was related to define Training Objectives, Training Elements and Training Targets. Other points of concern were related to the definition of scenarios and game changers.

\subsubsection{Objectives}

Setting up goals for a TORC training session was a challenging task. Although there are several documents explaining, it was difficult to develop a coherent set of objectives, targets and elements to guide a training preparation.

During the stages of preparation of our gameplay, it was observed the difficulty of establishing objectives to be trained, as well as the elaboration of the Training Elements and Training Targets. According to Grøtan, van der Vorm and Macchi [4], the first step is to define objectives, which, through elements, will refer to training targets. Because it was unclear how to elaborate these steps, the dynamic was designed over generic goals and without clear training Targets. This proved to be a difficult factor in the feedback stages of the rounds.

The clear definition of training objectives and targets is important in order to guide trainer, especially at the integrative moment between different groups. At this time, it is up to the trainer to guide the discussions of the participants to the points of interest to be trained.

Likewise, the clear definition of goals and targets should be used in structuring scenarios and game changers. Such elements of the game should allow participants to reflect on aspects of their organization that may imply in its objectives. In the case of the application, these elements were only elaborated based on research data, not deepening any specific topic, showing a point of fragility.

In short, a survey of critical points to be trained must be done in the organization and, therefore, objectives, elements and targets must be elaborated. The use of the pre-set objectives in the TORC reports did not prove to be effective since they were designed to specific application contexts where the dynamics was created and tested. Thus, the recommendation is that an effort to develop clear goals at the level of Resilience, Integrated Operations, System Response, Human Factors, non-technical skills, etc., should be done allowing for better chaining of sessions. 


\subsubsection{Game Definitions}

Trainer's preparation is essential in the application of TORC, trainer role is to ensure the achievement of objectives and leads each of the rounds of the game. The trainer has to be familiar with the principles of resilience, besides the gaming rules and this training method. We recommend inviting the trainer to be present in the preparation phase of training TORC, to get to know and to contribute to the scenarios that will be used in the training. The trainer can be external researcher or an employee from the company. We understand that when the trainer is a researcher, it is necessary to learn specific knowledge about the industry and organization who is participating in the TORC (e.g. technical language, routine expressions and operations knowledge). In our application, the trainers were researchers with a background in aviation, because it was considered that they would be more familiar with the scenarios studied, in the context of aerodromes.

Participants' selection should consider their work experience and their position in the company. Training people with more experience can bring positive points in relation to the quantity and quality of the stories, elements and targets of the training. On the other hand, composing a group with people from different backgrounds can serve as a form of knowledge transfer. The employee position also must be considered: whether the group will be composed of people of the same position (e.g. captains) or whether the group will be composed of people related to the same operation (e.g. crew of a flight: captain, first officer, flight attendants). People of the same occupation may share knowledge and experience relating to this activity; and people related to the same operation can share a systemic view.

Before the TORC application, there are many arrangements for the event, such as planning an agenda, preparing a room of the game and materials. Planning the agenda refers to establish all activities for the day training, especially limiting time for each moment of the application. Our application warned that two matches per day can be tiresome for the participants, especially because our participants were not familiar with TORC. Preparing the room concern to organize the physical spaces for the training. We recommend taking into account the size of the game board and other support materials that are needed. We ran the game in two groups at the same time in a room, due to the discussions and the conversations of each table, one group disturbed the other in some moments. For this reason, it is recommended that each group stay in a separate room, in addition to providing greater privacy, also creates a more conducive environment for the game. There are many materials for the game that have to be prepared, printed, so we recommend to use a checklist to make sure everything is under control.

\subsubsection{Context}

Scenarios selection was based on TORC materials, which recommended researchers to create training scenarios based on real events that happened in the organization being trained. It is important to advise that scenarios must be developed according to what is expected to be trained and that is must be adequate to the selected participants.

Our application showed that the scenarios could be further elaborated, with specific details on the situation. In their absence, participants were creating parameters (e.g. aircraft altitude, fatigue conditions of the involved, number of people in risk areas), which consequently made the groups' results different, even though each group was working in the same scenario. We recommend when designing a TORC game to take into account whether it is valid for the training to give more details to the scenario.

Game changers are the starting trigger for each round, which brings a change to the current scenario. The TORC method proposes six different types of game changers. For the application was created five game changers, one for each scheduled round, without defined typology. For the creation of game changers it was taken into account the ones that reflected 
critical factors in the original stories and that were independent from each other. In this way, the randomness could occur without the story losing its meaning.

In the first match, game changers were chosen randomly, while in the second it was sequentially organized in order to offer a greater difficulty each round. According to participants, game changers organized by degrees of difficulty ensured that the story was constructed with a temporal narrative. In the case of random game changers, although the cards were independent from each other, some informations made the group develop a narrative of potential consequences that was disrupted when a new card was not consistent with the story already constructed. In this case, participants reported that it was much more difficult to create different parallel histories and to remember which last version was created without being confused with the others.

\subsection{Application Phase}

This phase represents the gameplay. Tips in here concerns the training process (briefing, gameplay and debriefing) and the game format (operational, managerial and integrated).

\subsubsection{Training process}

Training starts with a Briefing which will present resilience concepts, training objectives and TORC method. It is important to make the participants familiar with resilience subjects covered in TORC game (e.g. sensemaking, situational awareness) and establish a theoretical leveling in order to get better use of the gaming dynamic. We recommend knowing the educational level of the participants and adjusting the introductory stage to level them. Our application showed that is may be interesting to create a interactive way of explaining the game, such as a video which shows how the game works or even to propose a simulation of the game.

Gameplay consists of four to six rounds and the moment of reflections by trainees and observers. We recommend being aware of time management, in order to keep a dynamic and productive discussion. Our applications shows us that the restriction of the maximum time per round may not lead to a time pressure, as desired. An alternative may be adding a new card when the time is finished and to create a pressure of new demands in a staggered way. We realize than the second gameplay of our application happened faster, because the participants knew how to play the TORC game.

Debriefing happens after gameplay, where it is reflected about the training experience and it is checked if the objectives were accomplished. The TORC method proposes a questionnaire to be completed by the participants at the end of the training session. Another version of the questionnaire must be filled out by the participant's manager sometime after the training to assess his or her perception of the employee's behavior changes. In our application participants were asked to answer the questionnaire and an open dialogue was created on the perceptions of the dynamics. However, it is recommended that it can be reflected on a closing dynamic that has a structured method for discussing the experience and evaluating it.

\subsubsection{Training format}

In relation to the training format, it is already discussed by Grøtan et al. [3] that a TORC training must encompass different levels of the organization. It is also pointed that hierarchical differences may be a problem if trainees fell inhibited to talk about organizational issues. In our application, there was a difference in flight experience between the two groups but not within group members, with one exception. The exception was in the group with most the experienced trainees, where there was one member considerably less experienced. This difference caused this trainee not to be comfortable in contributing. 


\subsection{Analyses' Phase}

In this phase, it is expected that trainers and managers discuss the implications and extract knowledge from the training session in order to enhance safety in operations. This analysis can be made in terms of relevance of the discussed topics related to resilience and management of critical situations; and in terms of the training method, in order to develop and adapt TORC to be replied in the organization.

\subsubsection{Discussion about the relevance}

A TORC training should not be limited to the gaming sessions. It is important to extract knowledge from the interaction between operators and managers in order to enhance the system, assuring better resources allocation and systemic knowledge. Some questions should be asked after a Training program: How this training has influenced operators and managers in understanding availability of resources and information in the system? What impact work of one team have in others? How system understand and respond to unforeseen ways of solving problems?

\subsection{Discussion about the training method}

TORC seems to offer a promising training method, even though it is necessary to discuss it utility and usability. Its utility refers to reflect about two questions: does it achieved its goal? Does it offer advantage over other training methods? Its usability refers if it is easy to apply.

\section{DISCUSSION AND CONCLUSIONS}

The objective of this article was to discuss a structure for TORC application. By means of literature review and an application of the game, it was possible to design an application framework that may help future research. This application had two groups of aviation pilots with different experiences. Discussions and questions to be answered in future applications of the dynamics were also raised. This work originated from a difficulty faced in elaborating a training based on TORC.

The proposed frame consists of three phases related to the main points to be considered in TORC game: development, application and analysis. Among the discussions raised during the elaboration of the Framework, three major topics deserve attention: preparation of the trainers; structure and game changers; and debriefing.

Preparation of trainers revealed the need for specific knowledge of the field of application, such as type of operation, jargon, technical terms, steps and activities, etc. At the same time, it is necessary to know about the concepts of resilience, in order to create the intended meaning of the game. Otherwise, the dynamics may end up taking different directions than expected, for example, it may turn TORC into game to activity execution training, or a game to find guilty and punish them.

Regarding the matches, it was noticed that the order of presentation of the game changers was an element of difficulty. Discussions on how to identify these, or even about the need for randomness can facilitate the game. Another point to be considered is the feasibility of creating a fault tree, where game changers will emerge according to the previous one, thus guaranteeing a sequentiality of the narrative. It was also noted the need for a greater structuring of the evaluation of learning and feedbacks, in order to generate organizational knowledge and to stimulate the replication of the tool.

The framework needs to be replicated in future research as a form of validation and refinement. Because it is based on a single application, the it still may not be fully representative. However, it represents a good starting point in attempting to develop a linear structure of the dynamics. 
In general, TORC demonstrates to be a valid tool in order to train resilience capacities in complex systems, because it allows the discussion of real problems. This reflexive process is capable of generating knowledge about the operation, systemic understanding of the impact of teamwork in the whole operation and understanding of the needs of resource availability.

\section{REFERENCES}

[1] K. E. Weick, Sensemaking in Organization. Sage, 1995.

[2] E. Hollnagel, C. P. Nemeth, and S. Dekker, Resilience Engineering Perspectives, Volume 2: Preparation and Restoration. Farnham: Ashgate, 2009.

[3] T. O. Grøtan et al., "Guidelines for the preparatory work needed to implement a TORC training program," Trondheim, 2016.

[4] T. O. Grøtan, J. van der Vorm, and L. Macchi, "Training for Operational Resilience Capabilities (TORC): 1st concepts elaboration," Trondheim, 2015.

[5] E. Hollnagel, D. D. Woods, and N. G. Leveson, Resilience Engineering: Concepts and Precepts. Ashgate Publishing Limited, 2006.

[6] T. O. Grøtan, "Hunting high and low for resilience: Sensitization from the contextual shadows of compliance," Safety, Reliab. Risk Anal. Beyond Horiz. - Proc. Eur. Saf. Reliab. Conf. ESREL 2013, no. February, pp. 327-334, 2014. 